\title{
Mutations at Nucleotide 1762, 1764 and 1766 of Hepatitis B Virus X Gene in Patients with Chronic Hepatitis B and Hepatitis B-Related Cirrhosis
}

\author{
Farzaneh Salarneia (BSc) \\ Department of Microbiology, School \\ of Medicine, Golestan university of \\ Medical Sciences, Gorgan, Iran \\ Sare Zhand (MSc) \\ Department of Microbiology, School \\ of Medicine, Golestan university of \\ Medical Sciences, Gorgan, Iran \\ Behnaz Khodabakhshi (MD) \\ Department of Infectious Disease, \\ School of Medicine, Iran university of \\ Medical Sciences, Tehran, Iran \\ Alijan Tabarraei (PhD) \\ Department of Microbiology, School \\ of Medicine, Golestan university of \\ Medical Sciences, Gorgan, Iran \\ Mohammad ali Vakili (PhD) \\ Department of Epidemiology, School \\ of Medicine, Iran university of Medical \\ Sciences, Tehran, Iran \\ Naeme Javid (MSc) \\ Department of Microbiology, School \\ of Medicine, Golestan university of \\ Medical Sciences, Gorgan, Iran \\ Masood Bazori (MSc) \\ Department of Microbiology, School \\ of Medicine, Golestan university of \\ Medical Sciences, Gorgan, Iran \\ Abdolvahab Moradi (PhD) \\ Department of Microbiology, School \\ of Medicine, Golestan university of \\ Medical Sciences, Gorgan, Iran \\ Corresponding author: Abdolvahab \\ Moradi \\ Email: abmoradi@yahoo.com \\ Tel: +989111772107
}

Address: Department of Microbiology, School of Medicine, Golestan university of Medical Sciences, Gorgan, Iran

Received : 19 Aug 2014

Revised: 07 Jan 2015

Accepted: 13 Jan 2015

\section{ABSTRACT}

Background and objective: Hepatitis B virus ( $\mathrm{HBV})$ is a DNA virus with high tendency toward hepatic tissue. There are currently about 3 million HBV-infected people and 350 to 400 million chronic carriers of this virus in the world. $X$ protein plays a role in the over-expression of oncogenes, carcinogenicity of liver cells and overlaps with the basal core promoter of the virus. Mutations at specific nucleotides of this region increase viral replication and liver disease progression. The aim of this study was to investigate the frequency of mutations at nucleotides 1762,1764 and 1766 of $\mathrm{HBV} X$ gene in patients with chronic hepatitis B and hepatitis B-related cirrhosis.

Methods: In this study, 102 patients including 68 chronic hepatitis patients and 34 patients with hepatitis B-related cirrhosis were enrolled. After DNA extraction, HBV X gene was amplified and sequenced using Semi Nested-PCR. Obtained gene sequences were compared with the standard sequence of $\mathrm{HBV}$ virus $X$ gene available in the gene bank (0kamoto AB033559). Then, the mutations in the gene $X$ of HBV were identified.

Results: Comparison of the standard sequence with sequences obtained from patients showed the presence of Al762T / G1764A mutation in 12 chronic (17.64\%) and 13 cirrhotic (30.23\%) patients. Also, C1766G / G1764T mutations were found in $8.23 \%$ of chronic patients and $17.64 \%$ of cirrhotic patients.

Conclusion: Al762T / G1764A mutations in the overlapping region of the basal core promoter with gene $X$ C-terminal may lead to liver disease progression from chronic hepatitis to cirrhosis, by changing the amino acid sequence of the $X$ protein.

Keywords: HBV; BCP Mutations; $X$ Gene Mutations; Cirrhosis; Chronic. 


\section{INTRODUCTION}

There are currently about 3 billion people infected with hepatitis B virus (HBV) and 350 to 400 million chronic carriers of this virus in the world $(1,2)$. Hepatitis is defined as the inflammation of the liver and HBV is one of the main causes of hepatitis (3). Nearly $3 \%$ of Iran's population are chronic carriers of $\mathrm{HBV}$ (4). HBV infection has a wide clinical spectrum ranging from chronic to acute hepatitis and cirrhosis to hepatic cancer (4). Mutations in the viral genome have a vital role in escalation of the infection. The circular DNA of the virus genome contains four open and almost overlapping transcription sites called S, C, P and X (5). X Protein (product of $X$ gene) plays a role in the overexpression of oncogenes and carcinogenesis of hepatic cells by activating signaling pathways. One of the regions which overlaps with the $\mathrm{X}$ gene in the genome of HBV (nucleotides 1374 to 1836), is the basal core promoter (BCP) (nucleotides 1742 to 1849$)(6)$. The overlapping region is the binding site of cellular and viral transcription factors thus, have a key role in regulating gene expression of the virus and host. Sequence change of this site is effective in increasing viral replication and progression of liver disease (7). Mutations of A1762T and G1764A nucleotides sites in the BCP, causes the conversion of Lys 130 Met and Val 131 I1e amino acids in the $\mathrm{X}$ protein. These mutations lead to decreased expression of P21, one of the most important regulators of cell cycle, by inhibiting P53 proto-oncogene (8). These mutations also create an appropriate site with tendency to bind to transcription factors of the hepatocyte nuclear factor 1 (HNF1) and thus, increase viral replication (9). It seems that these mutations can be used as prognostic factor of the disease progression. Based on occurrence of these mutations, patients with high risk of liver disease can be screened. The mentioned mutations can be also beneficial to improve the diagnostic methods and treatment strategies. The aim of this study was to evaluate the frequency of mutations at nucleotides 1762, 1764 and 1766 regions of $X$ gene of HBV, in two groups of patients with chronic hepatitis B and hepatitis B-related cirrhosis.

\section{MATERIAL AND METHODS}

This study (Ethics Committee code 9003170116) was performed on 102 patients infected with HBV consisted of 68 patients with chronic hepatitis B and 34 patients with cirrhosis, with confirmed diagnosis by a doctor using FibroScan. Approximately $5 \mathrm{ml}$ of blood were collected and plasma was separated by centrifugation at $3000 \mathrm{rpm}$ for 5 minutes. Then, $200 \mu$ of plasma was used for viral DNA extraction using High Pure Viral Nucleic Acid Kit (Roche Co.) according to the manufactures' instructions. To perform Semi Nested-PCR of the $\mathrm{X}$ gene at nucleotide positions 1374 to 1836 of the HBV genome, a pair of external primers and a pair of inner primers were used for the first and second rounds, respectively. First, 35 cycles were considered for the first round of the PCR program, including the initial step at temperature of $94{ }^{\circ} \mathrm{C}$ for denaturation of the double-stranded template for 1 minute, annealing temperature of $55{ }^{\circ} \mathrm{C}$ for $1 \mathrm{~min}$, extension at $72{ }^{\circ} \mathrm{C}$ for 1 minute and final elongation at $72{ }^{\circ} \mathrm{C}$ for 5 minutes. Then, the PCR products of the first round $(713 \mathrm{bp}$ fragment) were used for the second round. For the second round of the PCR, 35 cycles were considered, including the initial step at temperature of $94{ }^{\circ} \mathrm{C}$ for 5 minutes, denaturation at $94{ }^{\circ} \mathrm{C}$ for 30 seconds, annealing at $57{ }^{\circ} \mathrm{C}$ for 30 seconds, extension at $72{ }^{\circ} \mathrm{C}$ for 30 seconds and final elongation at $72{ }^{\circ} \mathrm{C}$ for 5 minutes. Then, the PCR products of the second round (516 bp fragment) was electrophoresed on $1.5 \%$ agarose gel and after staining with ethidium bromide was finally examined under UV light (Figure 1). The products were later sent for sequencing to the MacroGene Company, South Korea. In order to analyze and evaluate the mutations at the nucleotide level, Blast and Chromas softwares were used. The obtained sequences from the patients were compared with the standard Iranian HBV strains and standard reference $\mathrm{X}$ gene of $\mathrm{HBV}$, available in the GenBank (Okamoto AB033559). The results were analyzed by SPSS-16 using T-test and Chi-square analytical tests. P-value of less than 0.05 was considered as statistically significant. Data were reported as means \pm standard Deviations (SD) and as percentages, if necessary. Confidence interval (CI) of $95 \%$ was also calculated. 
Table 1- The primers used for Semi Nested-PCR (F: forward R: reverse)

\begin{tabular}{ccc}
\hline Primer & Sequence & Position \\
\hline F First round & ATCGTATCCATGGCTGCTAGGCT & $\mathbf{1 3 6 5 - 1 3 8 7}$ \\
R First round & CAGAATAGCTTGCCTGAGTGC & $\mathbf{2 0 5 8 - 2 0 7 8}$ \\
F Second round & ATCGTATCCATGGCTGCTAGGCT & $\mathbf{1 3 6 5 - 1 3 8 7}$ \\
R Second round & CACAGCTTGGAGGCTTGAACA & $\mathbf{1 8 6 1 - 1 8 8 1}$ \\
\hline
\end{tabular}

Figure 1-516bp band of positive samples next to the DNA Marker, the positive and negative controls

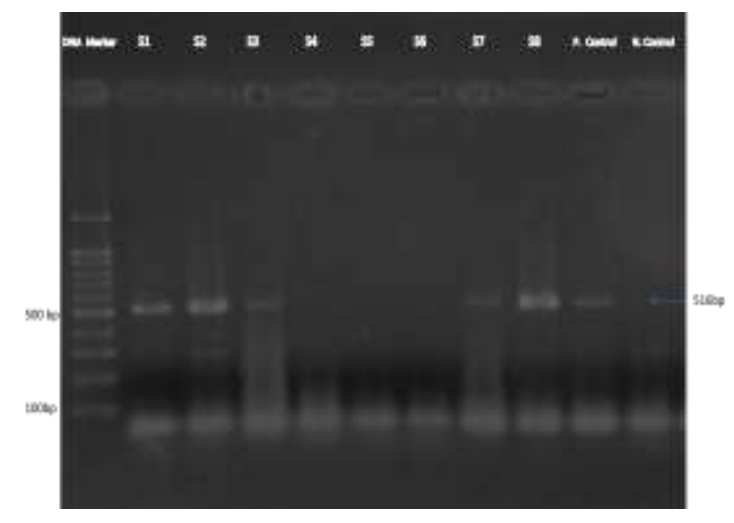

\section{RESULTS}

This study was performed on 68 patients with chronic hepatitis B including 17 females $(25 \%)$ and 51 males $(75 \%)$ and 34 patients with hepatitis B-related cirrhosis including seven females $(20.5 \%)$ and 27 males $(79.41 \%)$. The mean age of the subjects was $38.32 \pm 11.45$ years and $53.88 \pm 8.78$ years for the first and second group, respectively. There was a significant relationship between the parameter of age and disease stage between the two test groups $(\mathrm{P}=0.021)$. There was no significant relationship between gender and disease stage between the two groups of patients $(\mathrm{P}=0.620)$.

A1762T and G1764A mutations were observed simultaneously in 13 cirrhotic patients $(38.23 \%)$ and 12 patients (17.64\%) with chronic hepatitis $\mathrm{B}$. The simultaneous presence of G1764A/A1762T mutations in both groups showed a significant difference $(\mathrm{P}=0.023)$. G1764T mutation was observed in $20.50 \%$ of cirrhotic patients and $13.23 \%$ of patients with chronic hepatitis B. However, this mutation was not significantly different between the two groups $(\mathrm{P}=0.063)$. $\mathrm{C} 1766 \mathrm{G} / \mathrm{G} 1764 \mathrm{~T}$ mutations were observed simultaneously in six cirrhotic patients (17.64\%) and six patients with chronic hepatitis B $(8.82 \%)$. The simultaneous presence of this mutation showed no significant difference between the two groups.

\section{DISCUSSION}

Overall, the average age of cirrhotic patients was more than the average age of chronic patients, thus the index of age has a significant relationship with progression of the disease. In this study, the frequency of nucleotide 1762, 1764 and 1766 mutations at carboxylic end of $\mathrm{X}$ gene was compared between the two groups of patients with chronic hepatitis B and HBV-related cirrhosis. The most common mutations of the overlapping region of $\mathrm{C}$-terminal of $\mathrm{X}$ gene with BCP were G1764A/A1762T, G1764T and C1766G/G1764T mutations. A1762T and G1764A double mutations in HBV, at nucleotide positions A1762T and G1764A in the $\mathrm{X}$ gene-overlapping region with $\mathrm{BCP}$, were more frequent in cirrhotic patients compared to patients with mild liver disease. Products of these mutations were the transforming of Lys to Met at region 130 and Val to I1e at position 131 of the $X$ protein. This mutation decrease the expression of $\mathrm{P} 21$, as one of the most important regulators of cell cycle, by inhibiting the P53 proto-oncogene Hodgson et al. study in 2004, showed that in uninfected cells, active P53 protein increase the transcription of P21 gene (inhibitor of cyclin-dependent kinase). Given that the P21 protein inhibits the function of G1/S cyclins, the cell stops at the G1 phase. Thus, in infected liver cells, P53 protein is deactivated by the $\mathrm{X}$ protein and activates the $\mathrm{E} / \mathrm{Cdk} 2$ cyclin by reducing the $\mathrm{P} 21$ protein expression, which ultimately leads to passage of cell from phase G1 to $\mathrm{S}$ (10). This process leads to uncontrolled proliferation of HBVinfected hepatocytes. 
In this study, the double mutation of A1762T / G1764A was more frequent in cirrhotic patients (38.23\%) compared to patients with chronic hepatitis B (17.64\%). The occurrence of this mutation has an important role in liver disease progression to more severe stages. Many previous studies confirm the result of this study, including study of Chen et al. in China on patients with chronic hepatitis, cirrhosis and hepatocellular carcinoma (HCC) with frequency of $33 \%, 56 \%$ and $85 \%$, respectively (11). This study is also consistent with the study of Veazjalali et al. in Iran and Malki et al. in India that showed that increased frequency of A1762T / G1764A mutations increase the rate of disease progression. The frequency of this mutation in study of Veazjalali was $9 \%$ and $28 \%$ for patients with chronic hepatitis and cirrhotic patients, respectively. Malki et al study reported frequency of $33.6 \%, 46.7 \%$ and $66.7 \%$ for patients with chronic hepatitis, cirrhosis and HCC, respectively $(12,13)$. In addition to adenine to guanine nucleotide change at position 1764 of $\mathrm{X}$ gene, guanine to thymine nucleotide changes was also observed. The result of G1764T mutation at position 131 of the $\mathrm{X}$ protein is Valine to Leucine amino acid change. Concurrency of this mutation with mutation $\mathrm{C} 1766 \mathrm{G}$ at codon 131, leads to Valine to Isoleucine change at this position. This change in the $\mathrm{X}$ protein creates an appropriate binding site with more tendency to

\section{REFERENCES}

1. Khan A, Al Balwi MA, Tanaka Y, Hajeer A, Sanai FM, Al Abdulkarim I, et al. Novel point mutations and mutational complexes in the enhancer II, core promoter and precore regions of hepatitis $B$ virus genotype DI associated with hepatocellular carcinoma in Saudi Arabia. International Journal of Cancer. 2013;133(12): 2864-71.

2. Leupin O, Bontron S, Schaeffer C, Strubin M. Hepatitis $B$ virus $X$ protein stimulates viral genome replication via a DDB1-dependent pathway distinct from that leading to cell death. Journal of virology. 2005; 79(7): 4238-45.

3. Seeger C, Mason WS. Hepatitis B virus biology. Microbiology and Molecular Biology Reviews. 2000; 64(1): 51-68.

4. Merat S, Rezvan H, Nouraie M, Jamali A, Assari S, Abolghasemi $\mathrm{H}$, et al. The prevalence of hepatitis $B$ surface antigen and anti-hepatitis $B$ core antibody in Iran: a population-based study. Arch Iran Med. 2009; 12(3): 225-31. bind to HNF3 and subsequently leads to increased viral replication. Frequency of G1764T/C1766G in the cirrhotic group was more than the group of chronic patients, which indicates the possible role of this mutation in the development of disease from the chronic stage to liver cirrhosis. In agreement with the present study, Poustchi et al. study in Iran on HBV genotype D, showed that the frequency of G1764T / C1766G double mutations in the patients with cirrhosis and patients with chronic hepatitis B are $75 \%$ and $42 \%$, respectively. These results indicate the high frequency of this mutation (14).

\section{CONCLUSION}

A1762T / G1764A mutations in the overlapping region of the $\mathrm{X}$ gene $\mathrm{C}$-terminal with $\mathrm{BCP}$, leads to disease progression from the chronic stage to cirrhosis, by changing the amino acid sequence of the $X$ protein. It is recommended to study the effect of mutations at overlapping region of the $\mathrm{X}$ gene with $\mathrm{BCP}$ on other stages of $\mathrm{HBV}$ infection such as HCC.

\section{ACKNOWLEDGMENT}

The present study was derived from a research project for completion of MSc course in Virology. The authors would like to thank the Deputy of Research and Technology of Golestan University of Medical Sciences for their financial support.

\section{CONFLICT OF INTEREST}

Therer are no conflicts of interest.

5. Locarnini S, Zoulim F. Molecular genetics of $H B V$ infection. Antivir Ther. 2010;15(Suppl 3):3-14.

6. Cho EY, Choi CS, Cho J-H, Kim HC. Association between hepatitis $B$ virus $X$ gene mutations and clinical status in patients with chronic hepatitis $B$ infection. Gut and liver. 2011; 5(1): 70-6.

7. Wei Y, Tiollais P. Molecular biology of hepatitis B virus. Clinics in Liver Disease. 1999; 3(2): 189-219.

8. Kwun HJ, Jang KL. Natural variants of hepatitis $B$ virus $X$ protein have differential effects on the expression of cyclin-dependent kinase inhibitor p21 gene. Nucleic acids research. 2004; 32(7): 2202-13.

9. Mohammadkhani A, Montazeri G, Poustchi H. The Importance of Hepatitis B Virus Genome Diversity in Basal Core Promoter Region. Middle East Journal of Digestive Diseases (MEJDD). 2011; 3(1): 13-9.

10. Hodgson AJ, Keasler VV, Slagle BL. Premature cell cycle entry induced by hepatitis $B$ virus regulatory $H B x$ protein during compensatory liver regeneration. Cancer research. 2008; 68(24): 10341-8. 
11. Chen C-H, Lee C-M, Lu S-N, Changchien C-S, Eng $\mathrm{H}-\mathrm{L}$, Huang C-M, et al. Clinical significance of hepatitis $B$ virus (HBV) genotypes and precore and core promoter mutations affecting $H B V$ e antigen expression in Taiwan. Journal of clinical microbiology. 2005; 43(12): 6000-6.

12. Veazjalali M, Norder H, Magnius L, Jazayeri S, Alavian S, Mokhtari-Azad T. A new core promoter mutation and premature stop codon in the $S$ gene in $H B V$ strains from Iranian patients with cirrhosis. Journal of viral hepatitis. 2009; 16(4): 259-64.
13. Malik A, Singhal DK, Albanyan A, Husain SA, Kar P. Hepatitis $B$ virus gene mutations in liver diseases: a report from New Delhi. PloS one. 2012; 7(6): e39028.

14. Poustchi H, Mohamadkhani A, Bowden S, Montazeri G, Ayres A, Revill P, et al. Clinical significance of precore and core promoter mutations in genotype $D$ hepatitis $B$-related chronic liver disease. Journal of viral hepatitis. 2008; 15(10): 753-60. 